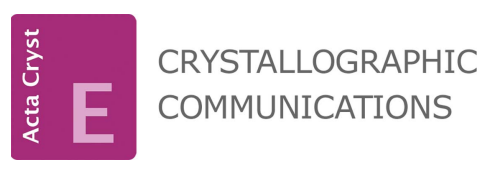

ISSN 2056-9890

Received 10 October 2017

Accepted 11 October 2017

Edited by P. C. Healy, Griffith University, Australia

₹ Additional correspondence author, e-mail: edwardt@sunway.edu.my.

Keywords: crystal structure; 1,2,3-triazole; Hirshfeld surface analysis.

CCDC reference: 1579464

Supporting information: this article has supporting information at journals.iucr.org/e

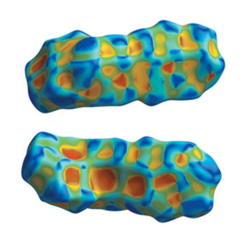

\section{4-Benzyl-1-(4-nitrophenyl)-1H-1,2,3-triazole: crystal structure and Hirshfeld analysis}

\author{
Julio Zukerman-Schpector, ${ }^{\mathrm{a} *}$ Sofia Dallasta Pedroso, ${ }^{\mathrm{a}}$ Lucas Sousa Madureira, ${ }^{\mathrm{a}}$ \\ Márcio Weber Paixão, ${ }^{\mathrm{b}}$ Akbar Ali ${ }^{\mathrm{b}}$ and Edward R. T. Tiekink ${ }^{\mathrm{c}} \ddagger$
}

\begin{abstract}
aLaboratório de Cristalografia, Esterodinâmica e, Modelagem Molecular, Departamento de Química, Universidade Federal de São Carlos, 13565-905 São Carlos, SP, Brazil, 'b Departamento de Química, Universidade Federal de São Carlos, 13565-905 São Carlos, SP, Brazil, and ${ }^{\mathbf{c}}$ Research Centre for Crystalline Materials, School of Science and Technology, Sunway University, 47500 Bandar Sunway, Selangor Darul Ehsan, Malaysia. *Correspondence e-mail: julio@power.ufscar.br
\end{abstract}

The molecule in the title compound, $\mathrm{C}_{15} \mathrm{H}_{12} \mathrm{~N}_{4} \mathrm{O}_{2}$, has a twisted $L$-shape with the dihedral angle between the aromatic rings of the $\mathrm{N}$-bound benzene and C-bound benzyl groups being $70.60(9)^{\circ}$. The nitro group is co-planar with the benzene ring to which it is connected $\left[\mathrm{C}-\mathrm{C}-\mathrm{N}-\mathrm{O}\right.$ torsion angle $\left.=0.4(3)^{\circ}\right]$. The threedimensional packing is stabilized by a combination of methylene-C-

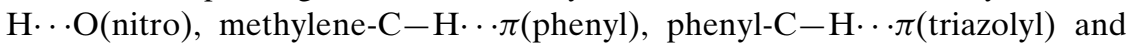
nitro-O $\cdots \pi$ (nitrobenzene) interactions, along with weak $\pi$ (triazolyl) $-\pi$ (nitrobenzene) contacts [inter-centroid distance $=3.8386$ (10) $\AA$ ]. The importance of the specified intermolecular contacts has been verified by an analysis of the calculated Hirshfeld surface.

\section{Chemical context}

The 1,2,3-triazoles comprise an important class of molecules, having a number of applications in biology and materials science. As reviewed recently, 1,2,3-triazoles display various potential pharmaceutical properties including anti-cancer, anti-viral, anti-tuberculosis and anti-microbial activities (Tron et al., 2008; Thirumurugan et al., 2013). The 1,2,3-triazole chromophore can function as a most useful scaffold in bioconjugation owing to its rigid framework, stability, and, crucially, water-solubility (Jewett \& Bertozzi, 2010; Holub \& Kirshenbaum, 2010). Further applications are known in the fields of dyes, photostabilizers and as agrochemicals (Golas \& Matyjaszewski, 2010; Qin et al., 2010). Very recently, a new and efficient synthesis for a metal-free and regioselective synthesis of 1,4-disubstituted 1,2,3-triazoles was described (Ali et al., 2014). Among the compounds synthesized in that study was the title compound, (I). Herein, the crystal and molecular structures of (I) are described along with an analysis of the Hirshfeld surface.

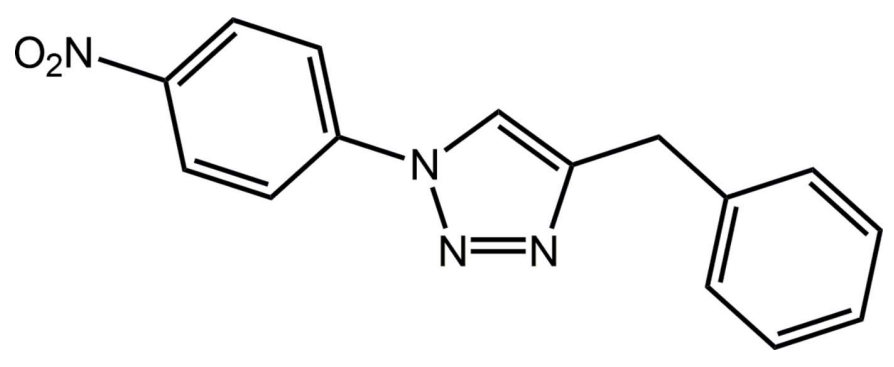


Table 1

Hydrogen-bond geometry $\left(\AA{ }^{\circ}\right)$.

Cg1-Cg3 are the centroids of the N1-N3,C1,C2, C4-C9 and C10-C15 rings, respectively.

\begin{tabular}{lllll}
\hline$D-\mathrm{H} \cdots A$ & $D-\mathrm{H}$ & $\mathrm{H} \cdots A$ & $D \cdots A$ & $D-\mathrm{H} \cdots A$ \\
\hline $\mathrm{C} 3-\mathrm{H} 3 B \cdots \mathrm{O} 2^{\mathrm{i}}$ & 0.97 & 2.58 & $3.452(3)$ & 150 \\
$\mathrm{C} 3-\mathrm{H} 3 A \cdots C 2^{\mathrm{ii}}$ & 0.97 & 2.96 & $3.857(2)$ & 154 \\
$\mathrm{C} 8-\mathrm{H} 8 \cdots C g 1^{\mathrm{iii}}$ & 0.93 & 2.86 & $3.665(3)$ & 146 \\
$\mathrm{~N} 4-\mathrm{O} 1 \cdots C g 3^{\text {iv }}$ & $1.21(1)$ & $3.67(1)$ & $4.1254(19)$ & $103(1)$ \\
\hline
\end{tabular}

Symmetry codes: (i) $-x+1,-y,-z+2$; (ii) $x-1, y, z$; (iii) $-x+2, y-\frac{1}{2},-z+\frac{3}{2}$; (iv) $-x+1,-y+1,-z+2$.

\section{Structural commentary}

The molecular structure of (I), Fig. 1, comprises a central, strictly planar 1,2,3-triazolyl ring (r.m.s. deviation of the five fitted atoms $=0.001 \AA$ ) flanked by $\mathrm{C}$ - and $\mathrm{N}$-bound benzyl and 4-nitrobenzene substituents, respectively. The dihedral angle between the five-membered ring and phenyl ring is $83.23(10)^{\circ}$, indicating a near perpendicular relationship. By contrast, the benzene ring is closer to co-planar to the triazolyl ring, forming a dihedral angle of $13.95(9)^{\circ}$. The dihedral angle between the outer rings is $70.60(9)^{\circ}$, indicating that the molecule has a skewed-shape based on the letter $L$. The nitro group is co-planar with the benzene ring to which it is bound as seen in the value of the $\mathrm{C} 12-\mathrm{C} 13-\mathrm{N} 4-\mathrm{O} 1$ torsion angle of $0.4(3)^{\circ}$.

\section{Supramolecular features}

The molecular packing of (I) features methylene-C$\mathrm{H} \cdots \mathrm{O}$ (nitro), methylene-C $-\mathrm{H} \cdots \pi$ (phenyl), phenyl-C$\mathrm{H} \cdots \pi($ triazolyl) and nitro-O $\cdots \pi$ (nitrobenzene) interactions, Table 1; the latter interactions have been described as being important in stabilizing the crystal packing of nitro-containing compounds (Huang et al., 2008). The $\mathrm{C}-\mathrm{H} \cdots \mathrm{O}$ and nitro$\mathrm{O} \cdots \pi$ interactions occur between centrosymmetrically related molecules while the $\mathrm{C}-\mathrm{H} \cdots \pi$ (phenyl) contacts occur along the $a$-axis direction and the $\mathrm{C}-\mathrm{H} \cdots \pi$ (triazolyl) contacts along the $b$-axis direction and, all taken together, consolidate the three-dimensional architecture, Fig. 2. Within the specified framework, weak $\pi$ (triazolyl) $-\pi$ (nitrobenzene $)^{\mathrm{i}}$ interactions occur with the inter-centroid distance $=3.8386(10) \AA$, interplanar angle $=13.95(9)^{\circ}$ for symmetry code: (i) $1-x,-y$, $2-z$.

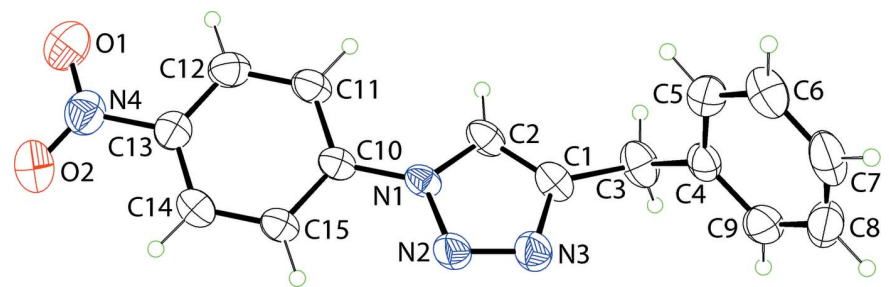

Figure 1

The molecular structure of (I), showing the atom-labelling scheme and displacement ellipsoids at the $35 \%$ probability level.

\section{Hirshfeld surface analysis}

The study of the Hirshfeld surface and intermolecular interactions of (I) has been carried out using standard parameters of the CrystalExplorer package (Wolff et al., 2012) and using similar protocols as in earlier studies (Zukerman-Schpector $e t$ al., 2017). In (I), the Hirshfeld surface is controlled by attractive interactions such as non-conventional $\mathrm{C}-\mathrm{H} \cdots \pi$, $\mathrm{C}-\mathrm{H} \cdots \mathrm{O}, \mathrm{C}-\mathrm{H} \cdots \mathrm{N}$ hydrogen bonds and $\pi-\pi$ interactions. The aforementioned contacts contribute around $70 \%$ to the overall surface area, Fig. 3 and Table 2. The repulsive $\mathrm{H} \cdots \mathrm{H}$ interactions account for the remaining $30 \%$, Fig. $3 b$. These observations may be rationalized in terms of the structure having electron-rich groups, i.e. the three aromatic rings and the nitro substituent, for which the electron densities are highly delocalized allowing them to have significant overlap in the molecular packing.

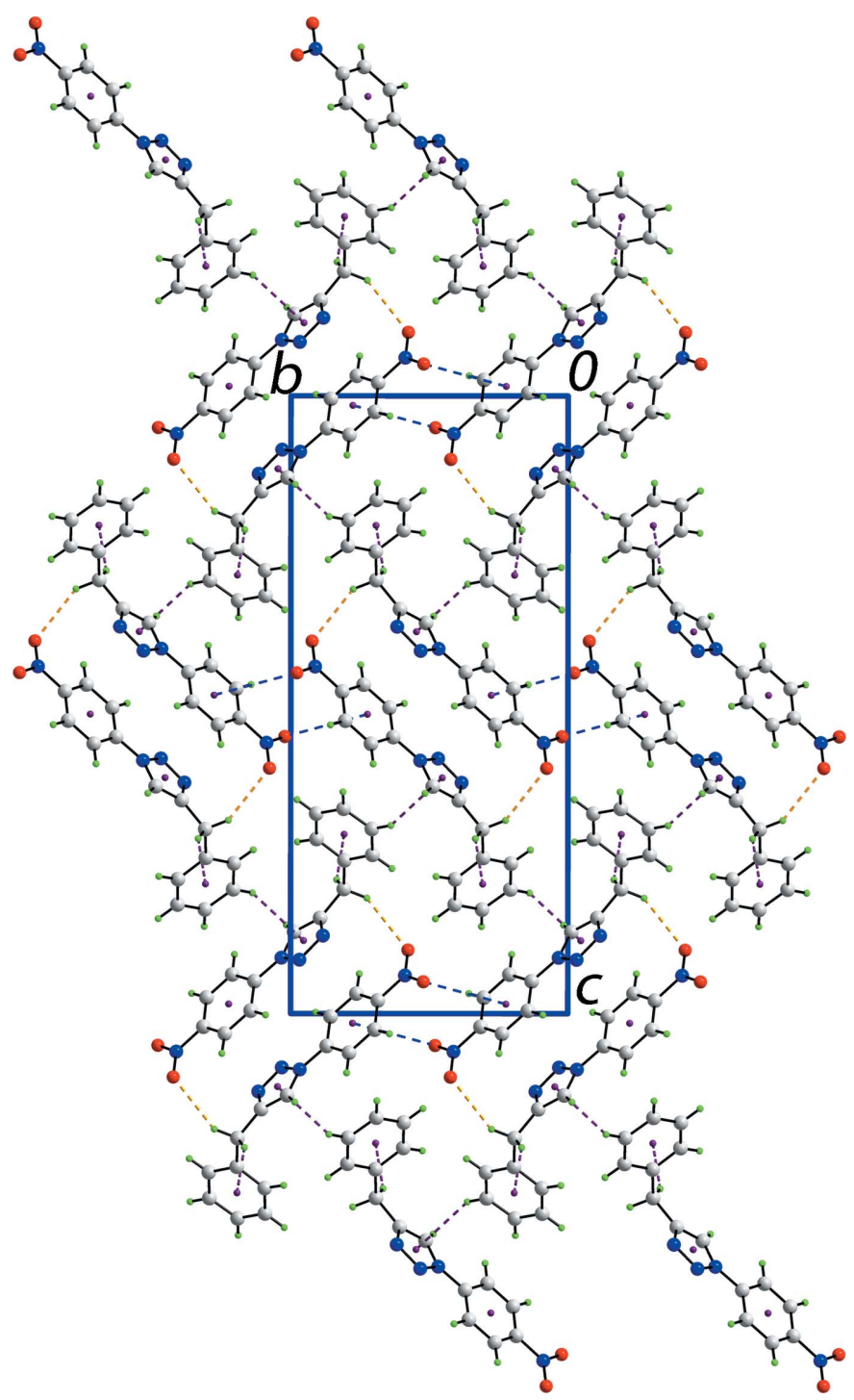

Figure 2

A view of the unit-cell contents in projection down the $a$ axis. The $\mathrm{C}-$ $\mathrm{H} \cdots \mathrm{O}, \mathrm{C}-\mathrm{H} \cdots \pi$ and nitro- $\mathrm{O} \cdots \pi$ contacts are shown as orange, purple and blue dashed lines, respectively. 
Table 2

Percentage contributions of interatomic contacts to the Hirshfeld surface for (I).

\begin{tabular}{ll}
\hline Contact & Percentage contribution \\
\hline $\mathrm{H} \cdots \mathrm{H}$ & 28.7 \\
$\mathrm{C} \cdots \mathrm{H} / \mathrm{H} \cdots \mathrm{C}$ & 26.1 \\
$\mathrm{O} \cdots \mathrm{H} / \mathrm{H} \cdots \mathrm{O}$ & 21.0 \\
$\mathrm{~N} \cdots \mathrm{H} / \mathrm{H} \cdots \mathrm{N}$ & 15.6 \\
$\mathrm{C} \cdots \mathrm{N} / \mathrm{N} \cdots \mathrm{C}$ & 3.9 \\
$\mathrm{C} \cdots \mathrm{O} / \mathrm{O} \cdots \mathrm{C}$ & 2.4 \\
others & 2.3 \\
\hline
\end{tabular}

(a)
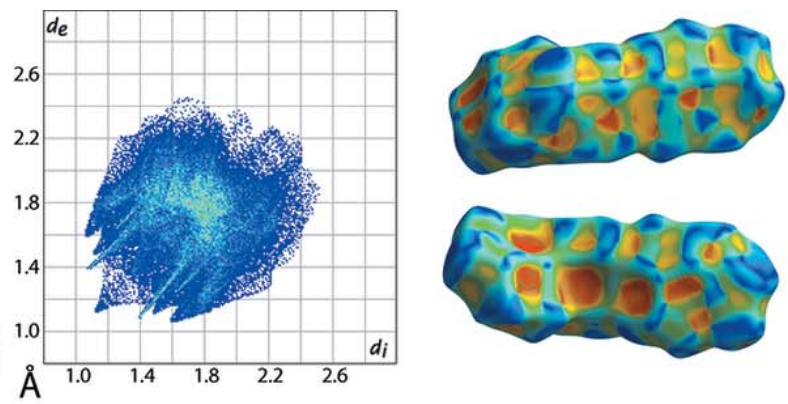

(c)
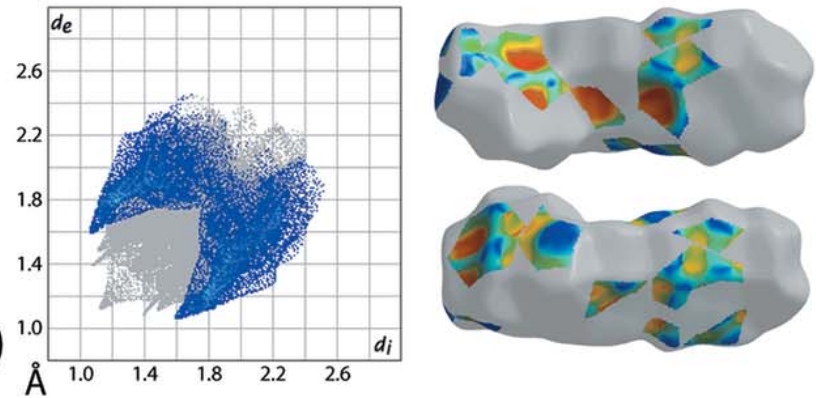

(e)
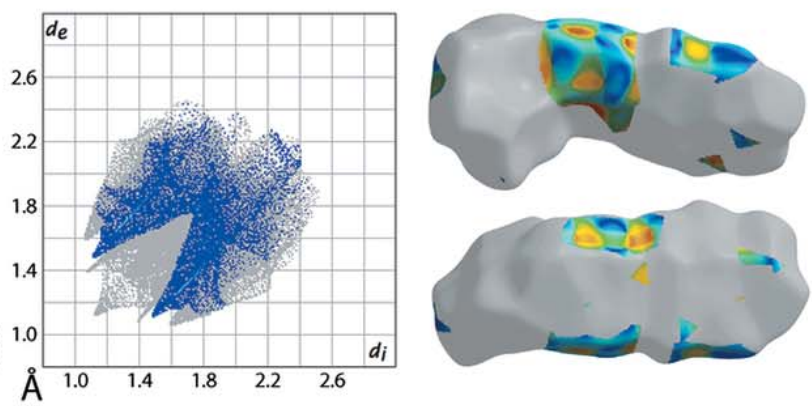

(b)
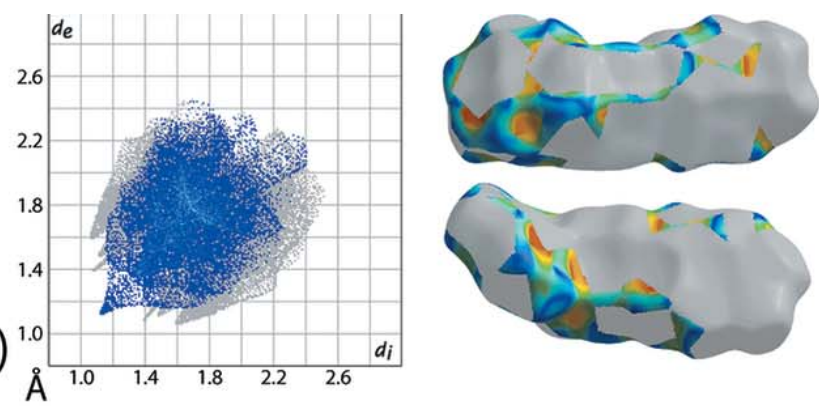

(d)
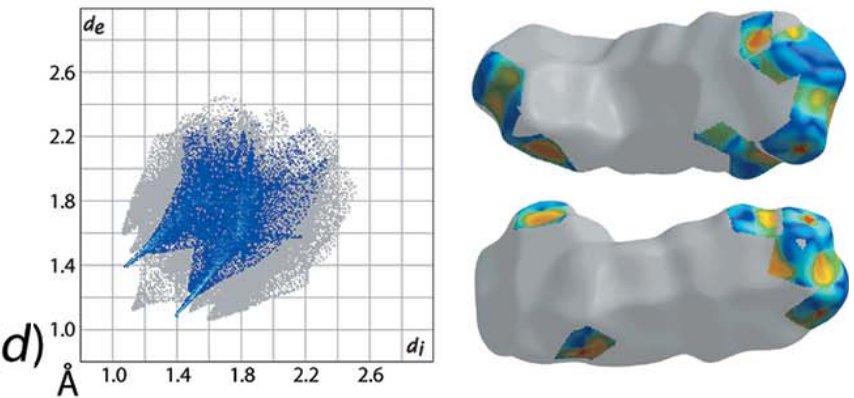

(f)
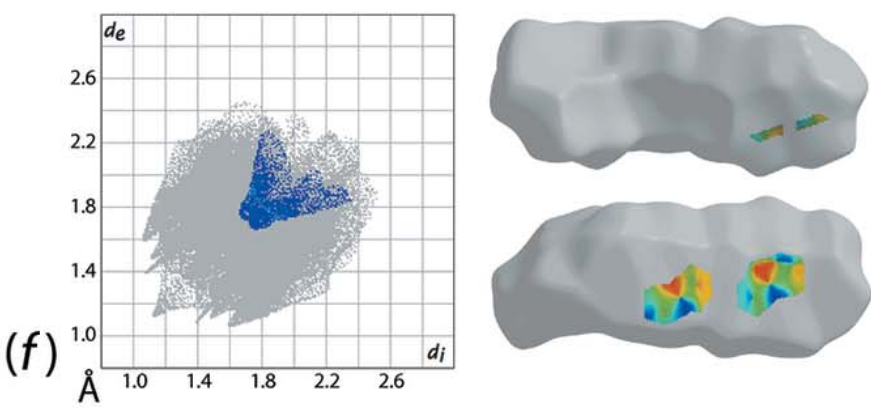

Figure 3
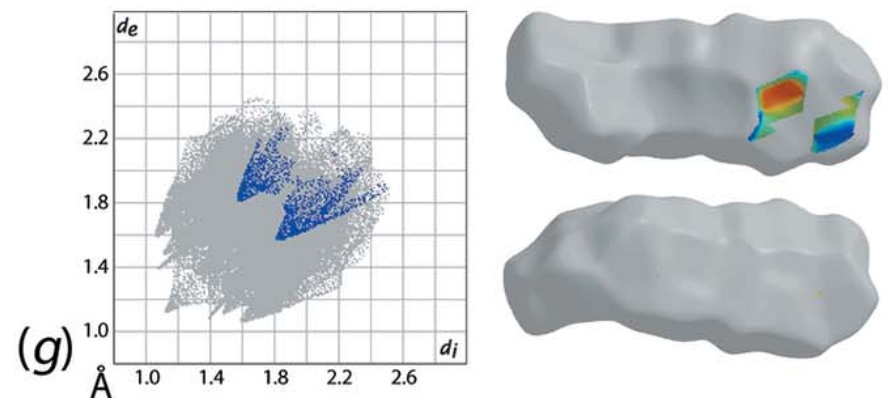

(a) The full two-dimensional fingerprint plot for (I) and two views of the Hirshfeld surface mapped over the shape-index property, and fingerprint plots delineated into $(b) \mathrm{H} \cdots \mathrm{H},(c) \mathrm{C} \cdots \mathrm{H} / \mathrm{H} \cdots \mathrm{C},(d) \mathrm{O} \cdots \mathrm{H} / \mathrm{H} \cdots \mathrm{O},(e) \mathrm{N} \cdots \mathrm{H} / \mathrm{H} \cdots \mathrm{N},(f) \mathrm{C} \cdots \mathrm{N} / \mathrm{N} \cdots \mathrm{C}$ and $(g) \mathrm{C} \cdots \mathrm{O} / \mathrm{O} \cdots \mathrm{C}$ interatomic contacts along with two views of Hirshfeld surface mapped over shape-index. 
As attractive interactions, the $\mathrm{C} \cdot \mathrm{H} / \mathrm{H} \cdots \mathrm{C}$ contacts contribute a significant role $(26.1 \%)$ to the overall surface area. These contacts arise mainly from $\mathrm{C}-\mathrm{H} \cdots \pi$ contacts spread over the entire molecule in which all rings, i.e. the triazole, nitrobenzene and benzyl rings, function as $\mathrm{H}$-atom acceptors, Tables 1 and 3, and Fig. 3c. The $\mathrm{O} \cdots \mathrm{H} / \mathrm{H} \cdots \mathrm{O}$ contacts contribute $21.0 \%$ to the Hirshfeld surface area. In essence, this arises owing to non-conventional $\mathrm{C}-\mathrm{H} \cdots \mathrm{O}$ hydrogen bonds, Fig. $3 d$. There are two different H-donor carbon atoms participating in the weak $\mathrm{C}-\mathrm{H} \cdots \mathrm{O}$ interactions, one of which is the methylene-C3 atom, Table 1, and the other being the nitrobenzene-C12 atom, Table 3. The $\mathrm{N} \cdots \mathrm{H} / \mathrm{H} \cdots \mathrm{N}$ contacts contribute approximately $16 \%$ to the overall surface area, Fig. $3 e$. Non conventional $\mathrm{C}-\mathrm{H} \cdots \mathrm{N}$ hydrogen bonds are formed with nitrobenzene-C atoms as $\mathrm{H}$ atom donors, Table 3 and Fig. $3 f$. The $\mathrm{C} \cdots \mathrm{N} / \mathrm{N} \cdots \mathrm{C}$ and $\mathrm{C} \cdots \mathrm{O} /$ $\mathrm{O}$. C contacts contribute around $6 \%$ to the Hirshfeld surface, Table 2 and Fig. $3 f$ and $g$. Other surface contacts do not contribute significantly to the molecular packing.

\section{Database survey}

There are only relatively few 1,2,3-triazole structures in the literature having $\mathrm{N}$-bound aryl groups and C-bound alkyl substituents. The two molecules closest to (I) have N-bound 4-chlorobenzene and C-bound $n$-butyl groups, i.e. (II) (Sarode et al., 2016), and N-bound 4-nitrobenzene and $C$-bound $n$-hexyl groups, i.e. (III) (Muhammad et al., 2015). In (II), the dihedral angle between the two planes is $22.59(7)^{\circ}$ and the $n$-butyl group is co-planar with the the five-membered ring as seen in the $s p^{2}-\mathrm{C}-\mathrm{C}_{\text {quaternary }}-\mathrm{C}-\mathrm{C}_{\text {methylene }}=0.06(4)^{\circ}$ and $\mathrm{C}_{\text {methylene }}-\mathrm{C}-\mathrm{C}-\mathrm{C}_{\text {methyl }}=-177.39(19)^{\circ}$ torsion angles. In (III), the aromatic rings are considerably more co-planar, $c f$. (I) and (II), with the dihedral angle between them being $2.65(8)^{\circ}$. With respect to the $n$-hexyl substituent, the structure of (III) resembles that of (I) in that the $s p^{2}-\mathrm{C}-\mathrm{C}_{\text {quaternary }}-$ $\mathrm{C}-\mathrm{C}_{\text {methylene }}$ torsion angle is $-118.4(3)^{\circ}$.

\section{Synthesis and crystallization}

The title compound was prepared as described in the literature (Ali et al., 2014). Crystals of (I) for the X-ray study were obtained by slow evaporation from an ethyl acetate $n$-hexane solution $(5: 1 v / v) .{ }^{1} \mathrm{H}$ NMR $\left(400 \mathrm{MHz}, \mathrm{CDCl}_{3}\right) \delta$ 7.70-7.65 (m, $2 \mathrm{H}), 7.59(\mathrm{~s}, 1 \mathrm{H}), 7.51-7.45(\mathrm{~m}, 2 \mathrm{H}), 7.42-7.32(m, 3 \mathrm{H}), 7.31-$ $7.21(m, 2 \mathrm{H}), 4.17(s, 2 \mathrm{H}) .{ }^{13} \mathrm{C} \mathrm{NMR}\left(100 \mathrm{MHz}, \mathrm{CDCl}_{3}\right) \delta=$ $148.5,138.8,137.2$, 129.6, 128.8, 128.7, 128.5, 126.6, 120.42, 119.6, 32.3 ppm. ESI-MS $(\mathrm{m} / z)$ calculated for $\mathrm{C}_{15} \mathrm{H}_{12} \mathrm{~N}_{4} \mathrm{O}_{2}$ $[M+\mathrm{H}]^{+} 281.1038$, found 281.1039 .

\section{Refinement details}

Crystal data, data collection and structure refinement details are summarized in Table 4. The carbon-bound $\mathrm{H}$ atoms were placed in calculated positions $(\mathrm{C}-\mathrm{H}=0.93-0.97 \AA)$ and were included in the refinement in the riding-model approximation, with $U_{\text {iso }}(\mathrm{H})$ set to $1.2 U_{\text {eq }}(\mathrm{C})$.
Table 4

Experimental details.

\begin{tabular}{|c|c|}
\hline \multicolumn{2}{|l|}{ Crystal data } \\
\hline Chemical formula & $\mathrm{C}_{15} \mathrm{H}_{12} \mathrm{~N}_{4} \mathrm{O}_{2}$ \\
\hline$M_{\mathrm{r}}$ & 280.29 \\
\hline Crystal system, space group & Monoclinic, $P 2_{1} / c$ \\
\hline Temperature $(\mathrm{K})$ & 293 \\
\hline$a, b, c(\AA)$ & $5.1962(1), 10.7814(3), 24.0067(6)$ \\
\hline$\beta\left({ }^{\circ}\right)$ & $90.256(2)$ \\
\hline$V\left(\mathrm{~A}^{3}\right)$ & $1344.90(6)$ \\
\hline$Z$ & 4 \\
\hline Radiation type & Мо $K \alpha$ \\
\hline$\mu\left(\mathrm{mm}^{-1}\right)$ & 0.10 \\
\hline Crystal size $(\mathrm{mm})$ & $0.46 \times 0.26 \times 0.14$ \\
\hline \multicolumn{2}{|l|}{ Data collection } \\
\hline Diffractometer & Bruker APEXII CCD \\
\hline Absorption correction & $\begin{array}{l}\text { Multi-scan (SADABS; Sheldrick, } \\
\text { 1996) }\end{array}$ \\
\hline$T_{\min }, T_{\max }$ & $0.695,0.745$ \\
\hline $\begin{array}{l}\text { No. of measured, independent and } \\
\text { observed }[I>2 \sigma(I)] \text { reflections }\end{array}$ & $9104,2450,1881$ \\
\hline$R_{\text {int }}$ & 0.023 \\
\hline$(\sin \theta / \lambda)_{\max }\left(\AA^{-1}\right)$ & 0.603 \\
\hline \multicolumn{2}{|l|}{ Refinement } \\
\hline$R\left[F^{2}>2 \sigma\left(F^{2}\right)\right], w R\left(F^{2}\right), S$ & $0.040,0.106,1.06$ \\
\hline No. of reflections & 2450 \\
\hline No. of parameters & 190 \\
\hline $\mathrm{H}$-atom treatment & $\mathrm{H}$-atom parameters constrained \\
\hline$\Delta \rho_{\max }, \Delta \rho_{\min }\left(\mathrm{e} \AA^{-3}\right)$ & $0.16,-0.18$ \\
\hline
\end{tabular}

Computer programs: APEX2 and SAINT (Bruker, 2009), SIR2014 (Burla et al., 2015), SHELXL2014 (Sheldrick, 2015), ORTEP-3 for Windows (Farrugia, 2012), DIAMOND (Brandenburg, 2006), MarvinSketch (ChemAxon, 2010) and publCIF (Westrip, 2010).

\section{Acknowledgements}

We thank Professor Regina H. A. Santos from IQSC-USP for the X-ray data collection.

\section{Funding information}

The Brazilian agency National Council for Scientific and Technological Development, CNPq, is gratefully acknowledged for fellowships to JZ-S (305626/2013-2) and MWP (13/ 02311-3). AA acknowledges CNPQ-TWAS for a scholarship.

\section{References}

Ali, A., Corrêa, A. G., Alves, D., Zukerman-Schpector, J., Westermann, B., Ferreira, M. A. B. \& Paixão, M. W. (2014). Chem. Commun. 50, 11926-11929.

Brandenburg, K. (2006). DIAMOND. Crystal Impact GbR, Bonn, Germany.

Bruker (2009). APEX2 and SAINT. Bruker AXS Inc., Madison, Wisconsin, USA.

Burla, M. C., Caliandro, R., Carrozzini, B., Cascarano, G. L., Cuocci, C., Giacovazzo, C., Mallamo, M., Mazzone, A. \& Polidori, G. (2015). J. Appl. Cryst. 48, 306-309.

ChemAxon (2010). Marvinsketch. http://www.chemaxon.com.

Farrugia, L. J. (2012). J. Appl. Cryst. 45, 849-854.

Golas, P. L. \& Matyjaszewski, K. (2010). Chem. Soc. Rev. 39, 13381354.

Holub, J. M. \& Kirshenbaum, K. (2010). Chem. Soc. Rev. 39, 13251337.

Huang, L., Massa, L. \& Karle, J. (2008). Proc. Natl Acad. Sci. 105, 13720-13723. 
Jewett, J. C. \& Bertozzi, C. R. (2010). Chem. Soc. Rev. 39, 1272-1279.

Muhammad, N. A., Khawaja, A. Y., Tariq, M., Fatima, W., Muhammad, H. K., Tahir, M. N., Safeena, Z. \& Shaista, A. (2015). Chin. J. Struct. Chem. 34, 1830-1840.

Qin, A., Lam, J. W. Y. \& Tang, B. Z. (2010). Chem. Soc. Rev. 39, 25222544.

Sarode, P. B., Bahekar, S. P. \& Chandak, H. S. (2016). Synlett, 27, 2681-2684.

Sheldrick, G. M. (1996). SADABS. University of Göttingen, Germany.

Sheldrick, G. M. (2015). Acta Cryst. C71, 3-8.
Thirumurugan, P., Matosiuk, D. \& Jozwiak, K. (2013). Chem. Rev. 113, 4905-4979.

Tron, G. C., Pirali, T., Billington, R. A., Canonico, P. L., Sorba, G. \& Genazzani, A. A. (2008). Med. Res. Rev. 28, 278-308.

Westrip, S. P. (2010). J. Appl. Cryst. 43, 920-925.

Wolff, S. K., Grimwood, D. J., McKinnon, J. J., Turner, M. J., Jayatilaka, D. \& Spackman, M. A. (2012). University of Western Australia.

Zukerman-Schpector, J., Prado, K. E., Name, L. L., Cella, R., Jotani, M. M. \& Tiekink, E. R. T. (2017). Acta Cryst. E73, 918-924. 


\section{supporting information}

Acta Cryst. (2017). E73, 1716-1720 [https://doi.org/10.1107/S2056989017014748]

\section{4-Benzyl-1-(4-nitrophenyl)-1H-1,2,3-triazole: crystal structure and Hirshfeld analysis}

Julio Zukerman-Schpector, Sofia Dallasta Pedroso, Lucas Sousa Madureira, Márcio Weber

\section{Paixão, Akbar Ali and Edward R. T. Tiekink}

Computing details

Data collection: APEX2 (Bruker, 2009); cell refinement: SAINT (Bruker, 2009); data reduction: SAINT (Bruker, 2009); program(s) used to solve structure: SIR2014 (Burla et al., 2015); program(s) used to refine structure: SHELXL2014 (Sheldrick, 2015); molecular graphics: ORTEP-3 for Windows (Farrugia, 2012) and DIAMOND (Brandenburg, 2006); software used to prepare material for publication: MarvinSketch (ChemAxon, 2010) and publCIF (Westrip, 2010).

4-Benzyl-1-(4-nitrophenyl)-1H-1,2,3-triazole

Crystal data

$\mathrm{C}_{15} \mathrm{H}_{12} \mathrm{~N}_{4} \mathrm{O}_{2}$

$M_{r}=280.29$

Monoclinic, $P 2_{1} / c$

$a=5.1962(1) \AA$

$b=10.7814(3) \AA$

$c=24.0067$ (6) $\AA$

$\beta=90.256(2)^{\circ}$

$V=1344.90(6) \AA^{3}$

$Z=4$

$F(000)=584$

Data collection

Bruker APEXII CCD

diffractometer

$\varphi$ and $\omega$ scans

Absorption correction: multi-scan

(SADABS; Sheldrick, 1996)

$T_{\min }=0.695, T_{\max }=0.745$

9104 measured reflections

Refinement

Refinement on $F^{2}$

Least-squares matrix: full

$R\left[F^{2}>2 \sigma\left(F^{2}\right)\right]=0.040$

$w R\left(F^{2}\right)=0.106$

$S=1.06$

2450 reflections

190 parameters

0 restraints
$D_{\mathrm{x}}=1.384 \mathrm{Mg} \mathrm{m}^{-3}$

Melting point $=371-373 \mathrm{~K}$

Mo $K \alpha$ radiation, $\lambda=0.71073 \AA$

Cell parameters from 2937 reflections

$\theta=3.2-25.0^{\circ}$

$\mu=0.10 \mathrm{~mm}^{-1}$

$T=293 \mathrm{~K}$

Irregular, yellow

$0.46 \times 0.26 \times 0.14 \mathrm{~mm}$

2450 independent reflections

1881 reflections with $I>2 \sigma(I)$

$R_{\text {int }}=0.023$

$\theta_{\max }=25.4^{\circ}, \theta_{\min }=1.7^{\circ}$

$h=-6 \rightarrow 6$

$k=-12 \rightarrow 12$

$l=-28 \rightarrow 28$

Hydrogen site location: inferred from neighbouring sites

$\mathrm{H}$-atom parameters constrained

$w=1 /\left[\sigma^{2}\left(F_{\mathrm{o}}^{2}\right)+(0.0383 P)^{2}+0.3766 P\right]$

where $P=\left(F_{\mathrm{o}}{ }^{2}+2 F_{\mathrm{c}}{ }^{2}\right) / 3$

$(\Delta / \sigma)_{\max }=0.001$

$\Delta \rho_{\max }=0.16 \mathrm{e} \AA^{-3}$

$\Delta \rho_{\min }=-0.18$ e $\AA^{-3}$ 


\section{Special details}

Geometry. All esds (except the esd in the dihedral angle between two 1.s. planes) are estimated using the full covariance matrix. The cell esds are taken into account individually in the estimation of esds in distances, angles and torsion angles; correlations between esds in cell parameters are only used when they are defined by crystal symmetry. An approximate (isotropic) treatment of cell esds is used for estimating esds involving l.s. planes.

Fractional atomic coordinates and isotropic or equivalent isotropic displacement parameters $\left(\AA^{2}\right)$

\begin{tabular}{|c|c|c|c|c|}
\hline & $x$ & $y$ & $z$ & $U_{\text {iso }} * / U_{\text {eq }}$ \\
\hline $\mathrm{C} 1$ & $0.6006(3)$ & $-0.10629(18)$ & $0.84523(7)$ & $0.0532(4)$ \\
\hline $\mathrm{C} 2$ & $0.4648(3)$ & $-0.01241(18)$ & $0.86767(6)$ & $0.0531(4)$ \\
\hline $\mathrm{H} 2$ & 0.3030 & 0.0154 & 0.8566 & $0.064 *$ \\
\hline $\mathrm{C} 3$ & $0.5294(4)$ & $-0.1916(2)$ & $0.79835(7)$ & $0.0672(5)$ \\
\hline $\mathrm{H} 3 \mathrm{~A}$ & 0.3644 & -0.1662 & 0.7832 & $0.081 *$ \\
\hline H3B & 0.5100 & -0.2750 & 0.8129 & $0.081 *$ \\
\hline $\mathrm{C} 4$ & $0.7242(3)$ & $-0.19387(16)$ & $0.75201(7)$ & 0.0519 (4) \\
\hline $\mathrm{C} 5$ & $0.7534(4)$ & $-0.09304(18)$ & $0.71743(8)$ & $0.0646(5)$ \\
\hline H5 & 0.6472 & -0.0244 & 0.7220 & $0.078^{*}$ \\
\hline C6 & 0.9359 (4) & $-0.0918(2)$ & $0.67636(8)$ & $0.0762(6)$ \\
\hline H6 & 0.9520 & -0.0224 & 0.6536 & $0.091 *$ \\
\hline $\mathrm{C} 7$ & $1.0933(4)$ & $-0.1905(3)$ & $0.66847(9)$ & $0.0803(7)$ \\
\hline $\mathrm{H} 7$ & 1.2195 & -0.1884 & 0.6411 & $0.096^{*}$ \\
\hline $\mathrm{C} 8$ & $1.0641(4)$ & $-0.2926(3)$ & $0.70110(10)$ & $0.0836(7)$ \\
\hline $\mathrm{H} 8$ & 1.1682 & -0.3616 & 0.6954 & $0.100 *$ \\
\hline $\mathrm{C} 9$ & $0.8791(4)$ & -0.29477 (19) & $0.74304(8)$ & $0.0695(5)$ \\
\hline H9 & 0.8607 & -0.3651 & 0.7651 & $0.083 *$ \\
\hline $\mathrm{C} 10$ & $0.5585(3)$ & $0.13033(15)$ & $0.94757(6)$ & 0.0444 (4) \\
\hline $\mathrm{C} 11$ & $0.3575(3)$ & $0.21075(18)$ & $0.93671(7)$ & $0.0577(5)$ \\
\hline H11 & 0.2615 & 0.2024 & 0.9041 & $0.069 *$ \\
\hline $\mathrm{C} 12$ & $0.2992(4)$ & $0.30319(18)$ & $0.97405(8)$ & $0.0627(5)$ \\
\hline H12 & 0.1625 & 0.3569 & 0.9674 & $0.075 *$ \\
\hline $\mathrm{C} 13$ & $0.4468(3)$ & $0.31461(16)$ & $1.02137(7)$ & $0.0541(4)$ \\
\hline $\mathrm{C} 14$ & $0.6508(3)$ & $0.23726(17)$ & $1.03240(7)$ & $0.0562(4)$ \\
\hline H14 & 0.7494 & 0.2478 & 1.0645 & $0.067 *$ \\
\hline $\mathrm{C} 15$ & $0.7071(3)$ & $0.14397(17)$ & $0.99526(6)$ & $0.0511(4)$ \\
\hline H15 & 0.8439 & 0.0904 & 1.0021 & $0.061 *$ \\
\hline N1 & $0.6118(2)$ & $0.03310(13)$ & $0.90952(5)$ & $0.0451(3)$ \\
\hline $\mathrm{N} 2$ & $0.8341(3)$ & $-0.03164(15)$ & $0.91281(6)$ & $0.0583(4)$ \\
\hline N3 & $0.8269(3)$ & $-0.11635(15)$ & $0.87374(6)$ & $0.0628(4)$ \\
\hline N4 & $0.3812(4)$ & $0.41242(16)$ & $1.06168(7)$ & $0.0729(5)$ \\
\hline $\mathrm{O} 1$ & $0.1984(4)$ & $0.47864(19)$ & $1.05159(8)$ & $0.1170(7)$ \\
\hline $\mathrm{O} 2$ & $0.5137(4)$ & $0.42327(16)$ & $1.10290(7)$ & $0.1031(6)$ \\
\hline
\end{tabular}

Atomic displacement parameters $\left(\AA^{2}\right)$

\begin{tabular}{lllllll}
\hline & $U^{11}$ & $U^{22}$ & $U^{33}$ & $U^{12}$ & $U^{13}$ & $U^{23}$ \\
\hline $\mathrm{C} 1$ & $0.0419(9)$ & $0.0744(12)$ & $0.0432(8)$ & $-0.0121(8)$ & $0.0010(7)$ & $0.0016(8)$ \\
$\mathrm{C} 2$ & $0.0367(8)$ & $0.0806(13)$ & $0.0420(8)$ & $-0.0047(8)$ & $-0.0035(7)$ & $0.0027(8)$
\end{tabular}




$\begin{array}{lllllll}\text { C3 } & 0.0609(11) & 0.0851(14) & 0.0556(10) & -0.0213(10) & 0.0005(9) & -0.0091(10) \\ \text { C4 } & 0.0513(10) & 0.0585(11) & 0.0458(8) & -0.0051(8) & -0.0084(7) & -0.0099(8) \\ \text { C5 } & 0.0728(13) & 0.0615(12) & 0.0596(11) & 0.0065(10) & 0.0030(9) & -0.0019(9) \\ \text { C6 } & 0.0879(15) & 0.0881(16) & 0.0526(11) & -0.0147(13) & 0.0046(10) & -0.0034(10) \\ \text { C7 } & 0.0646(13) & 0.122(2) & 0.0539(11) & -0.0053(14) & 0.0001(10) & -0.0310(13) \\ \text { C8 } & 0.0712(14) & 0.0994(18) & 0.0800(14) & 0.0278(13) & -0.0165(12) & -0.0410(14) \\ \text { C9 } & 0.0803(14) & 0.0621(12) & 0.0660(12) & 0.0053(11) & -0.0214(10) & -0.0076(10) \\ \text { C10 } & 0.0371(8) & 0.0573(10) & 0.0389(8) & -0.0030(7) & 0.0038(6) & 0.0098(7) \\ \text { C11 } & 0.0495(10) & 0.0753(13) & 0.0483(9) & 0.0074(9) & -0.0065(8) & 0.0056(9) \\ \text { C12 } & 0.0561(11) & 0.0688(12) & 0.0631(11) & 0.0122(9) & 0.0009(9) & 0.0083(9) \\ \text { C13 } & 0.0581(10) & 0.0533(10) & 0.0509(9) & -0.0034(8) & 0.0107(8) & 0.0048(8) \\ \text { C14 } & 0.0568(10) & 0.0665(11) & 0.0454(8) & -0.0074(9) & -0.0031(8) & 0.0035(8) \\ \text { C15 } & 0.0439(9) & 0.0627(11) & 0.0468(9) & 0.0007(8) & -0.0050(7) & 0.0048(8) \\ \text { N1 } & 0.0337(7) & 0.0630(9) & 0.0384(6) & 0.0005(6) & -0.0008(5) & 0.0050(6) \\ \text { N2 } & 0.0415(8) & 0.0786(10) & 0.0547(8) & 0.0099(7) & -0.0091(6) & -0.0088(8) \\ \text { N3 } & 0.0518(9) & 0.0784(11) & 0.0582(9) & 0.0064(8) & -0.0062(7) & -0.0122(8) \\ \text { N4 } & 0.0873(13) & 0.0647(11) & 0.0668(11) & -0.0013(10) & 0.0104(10) & -0.0005(9) \\ \text { O1 } & 0.1292(16) & 0.1118(14) & 0.1100(13) & 0.0544(13) & -0.0054(12) & -0.0279(11) \\ \text { O2 } & 0.1333(15) & 0.0942(12) & 0.0817(11) & 0.0065(11) & -0.0145(11) & -0.0274(9)\end{array}$

Geometric parameters $\left(\AA,{ }^{\circ}\right)$

\begin{tabular}{llll}
\hline $\mathrm{C} 1-\mathrm{C} 2$ & $1.347(2)$ & $\mathrm{C} 9-\mathrm{H} 9$ & 0.9300 \\
$\mathrm{C} 1-\mathrm{N} 3$ & $1.362(2)$ & $\mathrm{C} 10-\mathrm{C} 11$ & $1.382(2)$ \\
$\mathrm{C} 1-\mathrm{C} 3$ & $1.499(2)$ & $\mathrm{C} 10-\mathrm{C} 15$ & $1.386(2)$ \\
$\mathrm{C} 2-\mathrm{N} 1$ & $1.3516(19)$ & $\mathrm{C} 10-\mathrm{N} 1$ & $1.418(2)$ \\
$\mathrm{C} 2-\mathrm{H} 2$ & 0.9300 & $\mathrm{C} 11-\mathrm{C} 12$ & $1.375(3)$ \\
$\mathrm{C} 3-\mathrm{C} 4$ & $1.507(2)$ & $\mathrm{C} 11-\mathrm{H} 11$ & 0.9300 \\
$\mathrm{C} 3-\mathrm{H} 3 \mathrm{~A}$ & 0.9700 & $\mathrm{C} 12-\mathrm{C} 13$ & $1.373(2)$ \\
$\mathrm{C} 3-\mathrm{H} 3 \mathrm{~B}$ & 0.9700 & $\mathrm{C} 12-\mathrm{H} 12$ & 0.9300 \\
$\mathrm{C} 4-\mathrm{C} 9$ & $1.371(3)$ & $\mathrm{C} 13-\mathrm{C} 14$ & $1.374(2)$ \\
$\mathrm{C} 4-\mathrm{C} 5$ & $1.377(2)$ & $\mathrm{C} 13-\mathrm{N} 4$ & $1.472(2)$ \\
$\mathrm{C} 5-\mathrm{C} 6$ & $1.371(3)$ & $\mathrm{C} 14-\mathrm{C} 15$ & $1.376(2)$ \\
$\mathrm{C} 5-\mathrm{H} 5$ & 0.9300 & $\mathrm{C} 14-\mathrm{H} 14$ & 0.9300 \\
$\mathrm{C} 6-\mathrm{C} 7$ & $1.356(3)$ & $\mathrm{C} 15-\mathrm{H} 15$ & 1.9300 \\
$\mathrm{C} 6-\mathrm{H} 6$ & 0.9300 & $\mathrm{~N} 1-\mathrm{N} 2$ & $1.3516(18)$ \\
$\mathrm{C} 7-\mathrm{C} 8$ & $1.360(3)$ & $\mathrm{N} 2-\mathrm{N} 3$ & $1.209(2)$ \\
$\mathrm{C} 7-\mathrm{H} 7$ & 0.9300 & $\mathrm{~N} 4-\mathrm{O} 2$ & $1.212(2)$ \\
$\mathrm{C} 8-\mathrm{C} 9$ & $1.395(3)$ & $\mathrm{N} 4-\mathrm{O} 1$ & \\
$\mathrm{C} 8-\mathrm{H} 8$ & 0.9300 & & 119.8 \\
& & & 119.8 \\
$\mathrm{C} 2-\mathrm{C} 1-\mathrm{N} 3$ & $108.14(15)$ & $\mathrm{C} 4-\mathrm{C} 9-\mathrm{H} 9$ & $120.48(16)$ \\
$\mathrm{C} 2-\mathrm{C} 1-\mathrm{C} 3$ & $129.31(16)$ & $\mathrm{C} 8-\mathrm{C} 9-\mathrm{H} 9$ & $119.46(14)$ \\
$\mathrm{N} 3-\mathrm{C} 1-\mathrm{C} 3$ & $122.53(17)$ & $\mathrm{C} 11-\mathrm{C} 10-\mathrm{C} 15$ & $120.06(14)$ \\
$\mathrm{C} 1-\mathrm{C} 2-\mathrm{N} 1$ & $105.95(14)$ & $\mathrm{C} 11-\mathrm{C} 10-\mathrm{N} 1$ & $120.02(15)$ \\
$\mathrm{C} 1-\mathrm{C} 2-\mathrm{H} 2$ & 127.0 & $\mathrm{C} 15-\mathrm{C} 10-\mathrm{N} 1$ & 120.0 \\
$\mathrm{~N} 1-\mathrm{C} 2-\mathrm{H} 2$ & 127.0 & $\mathrm{C} 12-\mathrm{C} 11-\mathrm{C} 10$ & \\
$\mathrm{C} 1-\mathrm{C} 3-\mathrm{C} 4$ & $113.59(14)$ & $\mathrm{C} 12-\mathrm{C} 11-\mathrm{H} 11$ & \\
& & &
\end{tabular}




\begin{tabular}{|c|c|c|c|}
\hline $\mathrm{C} 1-\mathrm{C} 3-\mathrm{H} 3 \mathrm{~A}$ & 108.8 & $\mathrm{C} 10-\mathrm{C} 11-\mathrm{H} 11$ & 120.0 \\
\hline $\mathrm{C} 4-\mathrm{C} 3-\mathrm{H} 3 \mathrm{~A}$ & 108.8 & $\mathrm{C} 13-\mathrm{C} 12-\mathrm{C} 11$ & $118.71(17)$ \\
\hline $\mathrm{C} 1-\mathrm{C} 3-\mathrm{H} 3 \mathrm{~B}$ & 108.8 & $\mathrm{C} 13-\mathrm{C} 12-\mathrm{H} 12$ & 120.6 \\
\hline $\mathrm{C} 4-\mathrm{C} 3-\mathrm{H} 3 \mathrm{~B}$ & 108.8 & $\mathrm{C} 11-\mathrm{C} 12-\mathrm{H} 12$ & 120.6 \\
\hline $\mathrm{H} 3 \mathrm{~A}-\mathrm{C} 3-\mathrm{H} 3 \mathrm{~B}$ & 107.7 & $\mathrm{C} 12-\mathrm{C} 13-\mathrm{C} 14$ & $122.19(17)$ \\
\hline $\mathrm{C} 9-\mathrm{C} 4-\mathrm{C} 5$ & $117.75(18)$ & $\mathrm{C} 12-\mathrm{C} 13-\mathrm{N} 4$ & $118.55(17)$ \\
\hline $\mathrm{C} 9-\mathrm{C} 4-\mathrm{C} 3$ & $121.69(18)$ & $\mathrm{C} 14-\mathrm{C} 13-\mathrm{N} 4$ & $119.26(16)$ \\
\hline $\mathrm{C} 5-\mathrm{C} 4-\mathrm{C} 3$ & $120.56(17)$ & $\mathrm{C} 13-\mathrm{C} 14-\mathrm{C} 15$ & $119.00(16)$ \\
\hline $\mathrm{C} 6-\mathrm{C} 5-\mathrm{C} 4$ & $121.32(19)$ & $\mathrm{C} 13-\mathrm{C} 14-\mathrm{H} 14$ & 120.5 \\
\hline $\mathrm{C} 6-\mathrm{C} 5-\mathrm{H} 5$ & 119.3 & $\mathrm{C} 15-\mathrm{C} 14-\mathrm{H} 14$ & 120.5 \\
\hline $\mathrm{C} 4-\mathrm{C} 5-\mathrm{H} 5$ & 119.3 & $\mathrm{C} 14-\mathrm{C} 15-\mathrm{C} 10$ & $119.57(16)$ \\
\hline $\mathrm{C} 7-\mathrm{C} 6-\mathrm{C} 5$ & $120.8(2)$ & $\mathrm{C} 14-\mathrm{C} 15-\mathrm{H} 15$ & 120.2 \\
\hline $\mathrm{C} 7-\mathrm{C} 6-\mathrm{H} 6$ & 119.6 & $\mathrm{C} 10-\mathrm{C} 15-\mathrm{H} 15$ & 120.2 \\
\hline $\mathrm{C} 5-\mathrm{C} 6-\mathrm{H} 6$ & 119.6 & $\mathrm{C} 2-\mathrm{N} 1-\mathrm{N} 2$ & $109.62(14)$ \\
\hline $\mathrm{C} 6-\mathrm{C} 7-\mathrm{C} 8$ & $119.1(2)$ & $\mathrm{C} 2-\mathrm{N} 1-\mathrm{C} 10$ & $129.48(13)$ \\
\hline $\mathrm{C} 6-\mathrm{C} 7-\mathrm{H} 7$ & 120.5 & $\mathrm{~N} 2-\mathrm{N} 1-\mathrm{C} 10$ & $120.88(12)$ \\
\hline $\mathrm{C} 8-\mathrm{C} 7-\mathrm{H} 7$ & 120.5 & $\mathrm{~N} 3-\mathrm{N} 2-\mathrm{N} 1$ & $107.26(13)$ \\
\hline $\mathrm{C} 7-\mathrm{C} 8-\mathrm{C} 9$ & $120.5(2)$ & $\mathrm{N} 2-\mathrm{N} 3-\mathrm{C} 1$ & $109.03(15)$ \\
\hline $\mathrm{C} 7-\mathrm{C} 8-\mathrm{H} 8$ & 119.7 & $\mathrm{O} 2-\mathrm{N} 4-\mathrm{O} 1$ & $123.3(2)$ \\
\hline $\mathrm{C} 9-\mathrm{C} 8-\mathrm{H} 8$ & 119.7 & $\mathrm{O} 2-\mathrm{N} 4-\mathrm{C} 13$ & $118.35(19)$ \\
\hline $\mathrm{C} 4-\mathrm{C} 9-\mathrm{C} 8$ & $120.5(2)$ & $\mathrm{O} 1-\mathrm{N} 4-\mathrm{C} 13$ & $118.30(18)$ \\
\hline $\mathrm{N} 3-\mathrm{C} 1-\mathrm{C} 2-\mathrm{N} 1$ & $0.06(19)$ & $\mathrm{N} 4-\mathrm{C} 13-\mathrm{C} 14-\mathrm{C} 15$ & $178.33(15)$ \\
\hline $\mathrm{C} 3-\mathrm{C} 1-\mathrm{C} 2-\mathrm{N} 1$ & $178.62(16)$ & $\mathrm{C} 13-\mathrm{C} 14-\mathrm{C} 15-\mathrm{C} 10$ & $0.3(3)$ \\
\hline $\mathrm{C} 2-\mathrm{C} 1-\mathrm{C} 3-\mathrm{C} 4$ & $125.1(2)$ & $\mathrm{C} 11-\mathrm{C} 10-\mathrm{C} 15-\mathrm{C} 14$ & $1.0(2)$ \\
\hline $\mathrm{N} 3-\mathrm{C} 1-\mathrm{C} 3-\mathrm{C} 4$ & $-56.5(2)$ & $\mathrm{N} 1-\mathrm{C} 10-\mathrm{C} 15-\mathrm{C} 14$ & $-178.83(15)$ \\
\hline $\mathrm{C} 1-\mathrm{C} 3-\mathrm{C} 4-\mathrm{C} 9$ & $108.8(2)$ & $\mathrm{C} 1-\mathrm{C} 2-\mathrm{N} 1-\mathrm{N} 2$ & $-0.02(18)$ \\
\hline $\mathrm{C} 1-\mathrm{C} 3-\mathrm{C} 4-\mathrm{C} 5$ & $-70.5(2)$ & $\mathrm{C} 1-\mathrm{C} 2-\mathrm{N} 1-\mathrm{C} 10$ & $-178.32(15)$ \\
\hline $\mathrm{C} 9-\mathrm{C} 4-\mathrm{C} 5-\mathrm{C} 6$ & $-1.8(3)$ & $\mathrm{C} 11-\mathrm{C} 10-\mathrm{N} 1-\mathrm{C} 2$ & $-14.9(2)$ \\
\hline $\mathrm{C} 3-\mathrm{C} 4-\mathrm{C} 5-\mathrm{C} 6$ & $177.62(17)$ & $\mathrm{C} 15-\mathrm{C} 10-\mathrm{N} 1-\mathrm{C} 2$ & $164.92(16)$ \\
\hline $\mathrm{C} 4-\mathrm{C} 5-\mathrm{C} 6-\mathrm{C} 7$ & $0.2(3)$ & $\mathrm{C} 11-\mathrm{C} 10-\mathrm{N} 1-\mathrm{N} 2$ & $166.96(15)$ \\
\hline $\mathrm{C} 5-\mathrm{C} 6-\mathrm{C} 7-\mathrm{C} 8$ & $1.5(3)$ & $\mathrm{C} 15-\mathrm{C} 10-\mathrm{N} 1-\mathrm{N} 2$ & $-13.2(2)$ \\
\hline $\mathrm{C} 6-\mathrm{C} 7-\mathrm{C} 8-\mathrm{C} 9$ & $-1.6(3)$ & $\mathrm{C} 2-\mathrm{N} 1-\mathrm{N} 2-\mathrm{N} 3$ & $-0.03(18)$ \\
\hline $\mathrm{C} 5-\mathrm{C} 4-\mathrm{C} 9-\mathrm{C} 8$ & $1.7(3)$ & $\mathrm{C} 10-\mathrm{N} 1-\mathrm{N} 2-\mathrm{N} 3$ & $178.44(14)$ \\
\hline $\mathrm{C} 3-\mathrm{C} 4-\mathrm{C} 9-\mathrm{C} 8$ & $-177.68(16)$ & $\mathrm{N} 1-\mathrm{N} 2-\mathrm{N} 3-\mathrm{C} 1$ & $0.07(19)$ \\
\hline $\mathrm{C} 7-\mathrm{C} 8-\mathrm{C} 9-\mathrm{C} 4$ & $-0.1(3)$ & $\mathrm{C} 2-\mathrm{C} 1-\mathrm{N} 3-\mathrm{N} 2$ & $-0.1(2)$ \\
\hline $\mathrm{C} 15-\mathrm{C} 10-\mathrm{C} 11-\mathrm{C} 12$ & $-1.7(3)$ & $\mathrm{C} 3-\mathrm{C} 1-\mathrm{N} 3-\mathrm{N} 2$ & $-178.76(16)$ \\
\hline $\mathrm{N} 1-\mathrm{C} 10-\mathrm{C} 11-\mathrm{C} 12$ & $178.13(16)$ & $\mathrm{C} 12-\mathrm{C} 13-\mathrm{N} 4-\mathrm{O} 2$ & $-179.00(18)$ \\
\hline $\mathrm{C} 10-\mathrm{C} 11-\mathrm{C} 12-\mathrm{C} 13$ & $1.0(3)$ & $\mathrm{C} 14-\mathrm{C} 13-\mathrm{N} 4-\mathrm{O} 2$ & $1.6(3)$ \\
\hline $\mathrm{C} 11-\mathrm{C} 12-\mathrm{C} 13-\mathrm{C} 14$ & $0.3(3)$ & $\mathrm{C} 12-\mathrm{C} 13-\mathrm{N} 4-\mathrm{O} 1$ & $0.4(3)$ \\
\hline $\mathrm{C} 11-\mathrm{C} 12-\mathrm{C} 13-\mathrm{N} 4$ & $-179.03(16)$ & $\mathrm{C} 14-\mathrm{C} 13-\mathrm{N} 4-\mathrm{O} 1$ & $-178.99(19)$ \\
\hline $\mathrm{C} 12-\mathrm{C} 13-\mathrm{C} 14-\mathrm{C} 15$ & $-1.0(3)$ & & \\
\hline
\end{tabular}

Hydrogen-bond geometry $\left(A,{ }^{\circ}\right)$

$\mathrm{Cg} 1-\mathrm{Cg} 3$ are the centroids of the $\mathrm{N} 1-\mathrm{N} 3 / \mathrm{C} 1 / \mathrm{C} 2, \mathrm{C} 4-\mathrm{C} 9$ and $\mathrm{C} 10-\mathrm{C} 15$ rings, respectively.

\begin{tabular}{lllll}
\hline$D-\mathrm{H} \cdots A$ & $D-\mathrm{H}$ & $\mathrm{H} \cdots A$ & $D \cdots A$ & $D-\mathrm{H} \cdots A$ \\
\hline $\mathrm{C} 3-\mathrm{H} 3 B \cdots \mathrm{O} 2^{\mathrm{i}}$ & 0.97 & 2.58 & $3.452(3)$ & 150
\end{tabular}


supporting information

\begin{tabular}{lllll}
$\mathrm{C} 3-\mathrm{H} 3 A \cdots C g 2^{\mathrm{ii}}$ & 0.97 & 2.96 & $3.857(2)$ & 154 \\
$\mathrm{C} 8-\mathrm{H} 8 \cdots C g 1^{\mathrm{iii}}$ & 0.93 & 2.86 & $3.665(3)$ & 146 \\
$\mathrm{~N} 4-\mathrm{O} 1 \cdots C g 3^{\mathrm{iv}}$ & $1.21(1)$ & $3.67(1)$ & $4.1254(19)$ & $103(1)$ \\
\hline
\end{tabular}

Symmetry codes: (i) $-x+1,-y,-z+2$; (ii) $x-1, y, z$; (iii) $-x+2, y-1 / 2,-z+3 / 2$; (iv) $-x+1,-y+1,-z+2$. 